\title{
Blagovest Sendov - Pioneer of Mathematical Modeling in Bulgaria
}

\author{
Svetoslav Markov \\ Institute of Mathematics and Informatics-BAS \\ Acad. G. Bonchev st., block 8, 1113 Sofia, Bulgaria \\ Email: smarkov@bio.bas.bg
}

\section{My Teacher Prof. SEndov}

In February 2012 Prof. Blagovest Sendov turned 80. I first met him in 1963 as a student in mathematics at the Department of Physics and Mathematics of Sofia University. I was fascinated by his lectures in Numerical Methods. His first lecture was devoted to Mathematical modeling. On several realistic case studies Prof. Sendov revealed what was to me the philosophy of science. This lecture was crucial for my orientation in mathematical research. I was deeply impressed and I started to collect material for a book based on the ideas of Prof. Sendov, which I published later on [14]. During the years that followed, mathematical modeling became my main topic of interest. Thanks to Prof. Sendov, I was equipped with many useful ideas, tools and insights that I used in the study of real-life problems. Prof. Sendov's "philosophy" included a deep understanding of the mechanisms of the underlying real processes, the mathematical description of these processes using contemporary mathematical theories and the solution of the formulated mathematical problems using advanced numerical and computational tools.

An important area of mathematical applications is the one of biology. In the sequei I shall try to briefly survey Prof. Sendov's achievements in the field of mathematical modeling in biology and also mention his contributions to the field of "Interval analysis", which field is tightly related to Sendov's main topic of interest "Approximation theory" [25].

Prof. Sendov possesses the enormous ability to for- mulate difficult tasks and problems, playing a key role in mathematics and its applications, and requiring years of efforts to be resolved. At his weekly seminar on "Mathematical modeling" he used to pose such difficult problems and to make us young collaborators enthusiastic about working on them. He never pressed anybody of us to work on something particular, but he waited that everyone himself/herself chooses a topic of interest.

\section{The Contributions of Prof. Blagovest SENDOV TO BIOMATHEMATICS}

In the period 1965-1971 Prof. Sendov collaborated actively with Prof. Dr. Roumen Tsanev, an excellent molecular biologist, and also a very competent mathematician. In the summer of 1965 Prof. Sendov and Dr. Tsanev began jointly to study a hypothetical mechanisms for cellular proliferation, differentiation and carcinogenesis, suggested by Dr. Tsanev. The two scientists wanted to establish whether a mechanism for cellular activity based on interrelated genes is logically feasible. They decided to use the newly installed computer in the Institute of Mathematics at the Bulgarian Academy of Sciences to study a model of cellular activity based on a network of genes interrelated on the basis of equations describing the synthesis of mRNA, controlled by DNAprotein interactions and programming the ribosomes for the synthesis of proteins. During the next several years they had many discussions on the formulation of a suitable mathematical model. Prof. Sendov tried many formulations and performed multiple computer experiments. 


\section{S. Markov, Blagovest Sendov - Pioneer of Mathematical Modeling in Bulgaria}

The results of this active collaboration with lengthy discussions and computer experiments led to several modifications of the models, which were reported in a series of joint papers [1]-[10]. These papers are devoted to modeling of different biological objects such as epidermis and liver, or different processes such as cellular activity, cellular differentiation and carcinogenesis. The main result of the investigation was that the process has to be controlled by an independent carrier of genetic information, which is not necessarily semantically connected to the genetic information. This independent carrier of information was postulated as an epigenetic code. The mathematical model of living cells in a multi-cellular organism, based on the existence of an epigenetic code, was able to explain uniformly the processes of embryonic development, cytodifferentiation, vegetative reproduction, somatic embryogenesis, carcinogenesis and even the emergence of new forms of natural selection. All this is explained in [10] with more than 300 references to experimental results showing good agreement with the results produced by the mathematical model.

During the next several years a number of scientific papers are published, amongst them four papers in the Journal of Theoretical Biology, a survey paper in "Uspehi Mathematicheskih Nauk" and a monograph in Russian.

The studies of Sendov and Tsanev can be subdivided into four steps. The first step was to construct a mathematical model of living cells, based on the concept of Jacob and Monod for the existence of conjugate operons, working as a flip-flop. An operon has two states: repressed as inactive and derepressed as active. The mathematical realization of such flip-flop is by formulating a well-known set of nonlinear differential equations. The interaction between the different cells in an organism and the role of the nuclear membrane are important for eukaryotic cells. To this end a special variable for the diffusion through the membrane has been introduced. This variable depends on the functional state of the cell. It is known that when the eukaryotic cell enters in the mitotic cycle, the diffusion through its dissolved membrane stops. So they added to the system of differential equations, describing the activity of a cell, some additional differential equations with discontinuous right-hand side. The goal of this first model was to find out whether it is possible to choose the constants in the mathematical model of a system of synchronized cells, which divide and interact between themselves by the substances going through the membranes, in such a way that they reach a homeostasis. The result of the computer experiment is that the model is adequate under a suitable choice of the model parameters. The results obtained were in good agreement with the observed reaction of real tissues, such as the epidermal tissue, which was studied extensively by Dr Tsanev.

On the second stage a model of non-synchronized cells imitating the liver was constructed [11]. This model demonstrated a good agreement with experimental data, especially the reaction after a "partial hepatectomy". All this was achieved on the basis of the idea for repression and derepression.

The third stage was to model the mechanism of cytodifferentiation. Here the two scientists introduce the mechanisms of blocking and deblocking of the operons. In this situation, every operon has four different states: repressed-blocked, repressed-deblocked, derepressed-blocked and derepressed-deblocked. Only in the state derepressed-deblocked, the operon is active. To test these mechanisms, they constructed a mathematical model of a set of cells, which interacted between themselves. Every cell in the model has eight operons, one mitotic, responsible for the division of the cell, and seven functional operons. Every operon, when active, produces substances which may repress or deblock another operon. This interconnection between the operons is prescribed in the model by a matrix and represents the meaning of the epigenetic code. In other words, the existence of an epigenetic code means that the operons in a cell form a genetic network. Different types of cells in a organism have the same genetic information and differ by the set of blocked operons.

To define the interaction between the cells, a special geometrical arrangement of the cells has been proposed and the place of a new cell produced after division is prescribed. To make it simple, a population of cells called "Cylindros" has been created. To avoid the three dimensional geometry, an infinite cylinder is considered and a plane intersection of it is studied. Thus the Cylindros was a ring of two-dimensional cells on the plane with an interior space in the middle. The diffusion of all substances produced in the cells was possible only in the middle space and between the neighbor cells. In this way, the interaction between different cells was fulfilled. After division of a cell, the two new cells stayed on the same place on the ring as neighbors.

Choosing a particular matrix for the genetic net in the Cylindros, all important behaviors as embryonic development, cytodifferenciation, vegetative reproduction, somatic embryogenesis and carcinogenesis have been 


\section{S. Markov, Blagovest Sendov - Pioneer of Mathematical Modeling in Bulgaria}

demonstrated.

Mathematically the Cylindros was a system of ordinary differential equations of first order with discontinuous right hand sides and the number of the equations in this system depended on the time.

On the fourth stage of the study Prof. Sendov analyzes the system of ordinary differential equations for stability with respect to the number of the equations [11]-[12]. He shows that if the system is not stable, which means that the number of the equations goes to infinity, this case can be physiologically interpreted as cancerogenesis.

The collaborative work of Sendov and Tsanev is a typical example of tight interaction between the two sciences biology and mathematics. On one side biology benefits from mathematics, on the other side mathematics also benefits, as novel interesting problems and suitable tools for their solution appear. Such a tool is the analysis of Hausdorff-continuous functions and interval analysis. The contributions of Prof. Sendov to Hausdorff approximations are well known. Here I would like to mention some of his contributions to interval analysis.

\section{Novel Tools for Mathematical Modeling}

In many instances Sendov-Tsanev models make use of partially continuos functions of one variable, that is functions which are continuous in certain subintervals of their domain and have "jumps" in between. A typical such function is the Heaviside step function (function "jump") $s(x)=\{0, x<0 ; 1, x \geq 0\}$. Another famous function is the Dirac's function: $\delta(x)=\{0, x \neq 0 ; 1, x=0\}$. The interest of Prof. Sendov to such functions is well known. It can be assumed that it is this interests that led him to numerous results about these functions, and many scientific papers and books, in particular the integrated "Theory of Hausdorff Approximations" developed by him [25]. Sendov's approach to such functions is to consider their complete graphs by filling the jumps by intervals. Thus the complete graphs of the functions $s$ and $\delta$ are interval functions defined resp. as follows: $\bar{s}(x)=\{0, x<0 ;[0,1], x=0 ; 1, x>0\}, \bar{\delta}(x)=$ $\{0, x \neq 0 ;[0,1], x=0\}$. Then Sendov measures the distance between the complete graphs and the approximating function using the Hausdorff distance between the graphs as plane sets. A famous result obtained by prof Sendov in 1964 is that the Heaviside step function and the Dirac's function (and any bounded function in fact) can be approximated by algebraic polynomials of order $n$ in Hausdorff metric with accuracy: $C .(\ln n / n)$, $C=$ const. An important role in Sendov's theory of Hausdorff Approximations is played by the so-called
Hausdorff continuous functions, which are a special case of interval functions. In this way the theory of Hausdorff aproximations is tightly related to interval analysis.

Incidentally, the first difficult problem that was given to me by Prof. Sendov in my master thesis about finding a numerical algorithm for the Hausdorff approximation of the Heaviside step function using algebraic polynomials. At that time we performed numerical computations related to this problem using computer [13]. Many papers have been devoted to this problem, untill successfully solved [16]. The numerical approximation of functions like $s$ and $\delta$ still caue difficulties on contemporary computer platforms [15].

The investigations in the field of interval analysis were initiated by Prof. Sendov during 1976-1980. Interval functions have often been discussed in relation to Hausdorff approximations at Sendovs seminar on Approximation Theory held regularly at the Institute of Mathematics since 1964. Numerical computations related to the best polynomial Hausdorff approximations of certain interval functions require special attention to round-off errors. In 1975 Sendov, who was my PhD supervisor, gave me reprints of papers by T. Sunaga, H. Ratschek and G. Schroeder on interval arithmetic and differentiation of interval functions. I was very impressed by these papers, especially by the famous paper by T. Sunaga, which I studied thoroughly and later on I published (jointly with K. Okumura) a review of this extraordinary paper [17].

In the years to follow Prof. Sendov actively worked in the field of interval analysis and published several papers on the so-called S-limit and S-derivative of interval functions - terms that are tightly related to the theory of Hausdorff approximations, [18]-[20].

In this papers Sendov established a theory for analysis of interval functions. His studies have been continued by some of his many collaborators and during the years a lot of scientific papers have been published. Here I would like to mention some of the developments about the relations between Hausdorff continuous functions and interval analysis obtained in the past decade thanks to a new property established in 2004 by Roumen Anguelov.

The above mentioned novel property is that the set of Hausdorff-continuous functions is complete after Dedekind with respect to the familiar order relation. Let us note that the familiar functional spaces such as the space of continuous functions, the Sobolev spaces etc, with very few exceptions, are incomplete w.r.t. the order relation. Thus, a possibility appeared to solve a number of open problems in real analysis and the general theory of PDE or to improve previous results using 


\section{S. Markov, Blagovest Sendov - Pioneer of Mathematical Modeling in Bulgaria}

Hausdorff continuous functions. An important result is the introduction of algebraic operations with Hausdorffcontinuous functions and their application for numerical computations.

Let us mention that Hausdorff continuous functions form a special class of interval functions. It is wellknown that interval functions do not form a linear space. Thus it is an important fact that the algebraic operations for addition and multiplication by scalars in the set of continuous functions can be extended over the set of Hausdorff-continuous functions in such a way that they form a linear space. In several papers (jointly with Prof. Sendov) it has been shown that the space of Hausdorff continuous functions is the largest linear space of interval functions [21]-[23]. The obtained results have been applied to numerical computations [24].

\section{The CONFERENCE BIOMATH-2012}

During the years Prof. Sendov supported the biomathematical research in the Bulgarian Academy of Sciences. He co-chaired the first international conference BIOMATH 1995 [26] and took active part in many biomathematical workshops and seminars held at the Academy.

The present conference BIOMATH-2012 [27] proves that the pioneering work of Prof. Sendov in the field of mathematical modelling is alive. Our wish is the BIOMATH Conferences to become a forum for biologists and mathematicians, chemists and physicists, computer scientists and others, working together as partners in research connected with living organisms and the applications of the results to medicine, biology, ecology, agriculture and elsewhere. It is natural in such collaborations that the leading ideas come from the biologists. However, success depends on the abilities of both sides, especially when complicated mathematical models are involved.

In this article we tried to outline part of Prof. Sendov's activities related to Mathematical Modelling. The interested reader may find more about Prof Sendov's activities in the article [27] where also some of his contributions to Bulgarian education are presented in some detail.

Let us wish Prof. Sendov good health and still more future success in his scientific and social activities.

\section{REFERENCES}

[1] Tsanev, R. and Bl. Sendov: A model of the regulatory mechanism of cellular proliferation, C. r. Acad. Bulgare Sci., 19, (1966), no. 9, 835-838.
[2] Tsanev, R. and Bl. Sendov: A model of the regulatory mechanism of cellular multiplication, J. Theoret. Biol., New York, 12, (1966), 327-341.

[3] Sendov, Bl. and R. Tsanev: Modeling of the regulatory mechanism of the cellular proliferation in the liver, Central. Biochem. Lab. BAS, 3, (1968), 21-35 (in Bulgarian).

[4] Tsanev, R. and Bl. Sendov: Computer studies on the mechanism controlling cellular proliferation, in: Effects on radiation on cellular proliferation and differentiation. Vienna, Int. Atomic Energy Agency. 1968, 453-461.

[5] Sendov, B1. and R. Tsanev: Computer simulation of the regenerative processes in the liver, J. Theoret. Biol., New York, 18, (1968), 90-104.

[6] Sendov, Bl. and R. Tsanev: Computer simulation of the regulatory mechanisms of cellular proliferation, Inform. Processing, Amsterdam, 68 (1969), 1506-1507.

[7] Tsanev, R. and Bl. Sendov: A model of cancer studies by a computer, J. Theoret. Biol. 23, (1969), 124-134. http://dx.doi.org/10.1016/0022-5193(69)90071-X

[8] Tsanev, R. and Bl. Sendov: A possible mechanism for cellular differentiation, C. r. Acad. Bulgare Sci., 22, (1969), no. 12, 1433-1436.

[9] Sendov, B1., R. Tsanev and E. Mateeva: A mathematical model of the regulation of cellular proliferation of the epidermis, Izv. Math. Inst., BAS, 11, (1970), 221-246.

[10] Tsanev, R. and Bl. Sendov: Possible molecular mechanism for cell differentiation in multicellular organisms, J. Theoret. Biol., New York, 30, (1971), 337-193.

[11] Sendov, B1.: Mathematical models of the cellular proliferation and differentiation, Uspehi Math. Nauk, Moscow, 31 no. 3, (1976), 255-256 (in Russian).

[12] Sendov, B1.: Mathematical models of the processes for cellular proliferation and differentiation, Publ. Moscow University, 1976 (in Russian).

[13] Markov, S., Bl. Sendov, On the numerical computation of a class of polynomials of best approximation, Ann. Univ. Sofia, Math. Fac., 61, 1966/67, 17-27 (in Bulgarian).

[14] Markov, S., Mathematical Modeling, Nauka i Izkustvo, Sofia, 1977. (In Bulgarian).

[15] Dimitrova, N., S. Markov, Verified Computation of Fast Decreasing Polynomials, Reliable Computing 5 (3), 229-240, 1999.

http://dx.doi.org/10.1023/A:1009972120539

[16] Ivanov, K., V. Totik, Fast decreasing polynomials, Constructive Approximations 6 (1990), 1-20. http://dx.doi.org/10.1007/BF01891406

[17] Markov, S., K. Okumura, The contribution of T. Sunaga to interval analysis and reliable computing, In: Csendes, T. (Ed.), Developments in Reliable Computing, Kluwer, 1999, 167-188.

[18] Sendov, Bl., Segment arithmetic and segment limit. C. R. Acad. bulg. sci., 1977, 30, 955-968.

[19] Sendov, Bl., Segment derivatives and Taylor's formula. C. R. Acad. bulg. sci., 1977, 30, 1093-1096.

[20] Sendov, B1., Some topics of segment analysis. In: Interval Mathematics'80 (Ed. by K. Nickel), Academic Press, 1980, 236-245.

[21] Anguelov, R., S. Markov, Bl. Sendov, On the Normed Linear Space of Hausdorff Continuous Functions, Lecture Notes in Computer Science 3743, Springer, 2005, 281-288;

[22] Anguelov, R., S. Markov, B1. Sendov, The Set of Hausdorff Continuous Functions - the Largest Linear Space of Interval Functions, Reliable Computing 12 (2006), 337-363; 
[23] Anguelov, R., S. Markov, B1. Sendov, Algebraic operations on the space of Hausdorff continuous interval functions, In: B. D. Bojanov (Ed.), Constructive theory of functions, Varna 2005, Prof. Marin Drinov Academic Publ. House, 2006, 35-44.

[24] Anguelov, R., S. Markov, Numerical Computations with Hausdorff Continuous Functions, In: T. Boyanov et al. (Eds.), NMA 2006, Lecture Notes in Computer Science 4310, 2007, 279-286.

[25] Sendov, B1., Hausdorff Approximations, Kluwer, 1990. http://dx.doi.org/10.1007/978-94-009-0673-0

[26] http://www.biomath.bg/1995/BIOMATH95.php

[27] http://www.biomath.bg/2012

[28] Kenderov, P, A. Andreev, S. Dimova, S. Markov, Academician Blagovest Sendov at 80, In: J. Revalski (Ed.) Mathematics and Education in Mathematics, Sofia, UMB, 7-22 (In Bulgarian). 